RESEARCH ARTICLE

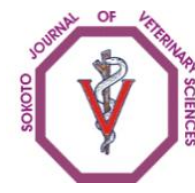

Sokoto Journal of Veterinary Sciences

(P-ISSN 1595-093X/ E-ISSN 2315-6201)

Oguntoye \& Oke /Sokoto Journal of Veterinary Sciences (2014) 12(3): 21-25

http://dx.doi.org/10.4314/sokjvs.v12i3.4

\title{
A Comparison of xylazine/ketamine, diazepam/ketamine and acepromazine/ketamine anaesthesia in Rabbit
}

\author{
CO Oguntoye* \& BO Oke
}

\author{
Faculty of Veterinary Medicine, \\ University of Ibadan
}

*Correspondence: Tel.: +2348055063671: E-mail: wumcel06@gmail.com

\begin{abstract}
The efficacy and safety of the recommended intramuscular (im) clinical doses of xylazine-ketamine (5mg$35 \mathrm{mg} / \mathrm{kg})$, diazepam-ketamine $(5 \mathrm{mg}-60 \mathrm{mg} / \mathrm{kg})$ and acepromazine-ketamine $(5 \mathrm{mg}-75 \mathrm{mg} / \mathrm{kg})$ post administration were obtained, evaluated and compared using the following parameters in 10 apparently healthy rabbits: vital parameters (means of heart rates, respiratory rates and temperature), onset of loss of righting reflex, onset of analgesia, duration of analgesia, duration at recumbency and attempts to stand. Onset of loss of righting reflex was not significantly different $(p>0.05)$ with xylazine-ketamine, diazepam-ketamine and acepromazine-ketamine at 3.4 $\pm 0.3,2.0 \pm 0.0$ and $2.6 \pm 0.3$ minutes respectively. Diazepam-ketamine and acepromazine-ketamine showed no analgesia while xylazine-ketamine had onset of analgesia and duration of analgesia of $4.2 \pm 0.1$ and $57.0 \pm 0.7$ minutes respectively. Duration of recumbency and first attempt to stand were longest with diazepam-ketamine, intermediate with acepromazine-ketamine and shortest with xylazine-ketamine of $184.4 \pm 3.3$ and 42.0 \pm 1.4 ; $169.9 \pm 3.0$ and $26.6 \pm 1.7$; and $62 \pm 1.5$ and $11.2 \pm 0.9$ minutes respectively. Mean heart rate ranged between $173.2 \pm 6.6$ to $187.2 \pm 7.5 ; 188.6 \pm 6.4$ to $206.0 \pm 3.1$ and $190.8 \pm 1.3$ to $202.8 \pm 1.9$ beats/minute with xylazine-ketamine, diazepam-ketamine, and acepromazine-ketamine respectively. Mean respiratory rate ranges were $58.4 \pm 3.5$ to $133.0 \pm 11.2 ; 71.4 \pm 6.9$ to $121.2 \pm 12.6$ and $80.8 \pm 6.2$ to $137.0 \pm 4.3$ breaths/minute with xylazine-ketamine, diazepamketamine and acepromazine-ketamine respectively. Mean temperature ranges were $39.9 \pm 0.1$ to $40.0 \pm 0.1$; $39.0 \pm 0.3$ to $39.9 \pm 0.1$ and $39.6 \pm 0.1$ to $40.2 \pm 0.0^{\circ} \mathrm{C}$ with xylazine-ketamine, diazepam-ketamine and acepromazineketamine respectively. In conclusion, all the three ketamine drug combinations produced unconsciousness and immobility, but only xylazine-ketamine provided additional analgesia. They were associated with normal heart rate and rectal temperature, as well as polypnoea of some clinical concern. Only xylazine-ketamine is potentially useful for painful procedures. Addition of specific analgesics to diazepam-ketamine and acepromazine-ketamine may make them useful for painful procedures.
\end{abstract}

Keywords: Acepromazine, Anaesthesia, Diazepam, Ketamine, Rabbit, Xylazine

Received: 20-02-2014

Accepted: 11-07-2014

\section{Introduction}

Considering that inhalant anaesthetic delivery apparatus can cause practical problems in the rabbit, injectable anaesthesia involving ketamine combinations is currently popular in this species (Flecknell, 2009). In this regard, ketamine is often combined with an alpha-2-agonist (e.g. xylazine), a benzodiazepine (e.g. diazepam) or a phenothiazine tranquilliser (e.g. acepromazine) to enhance muscle relaxation and analgesia and prolong duration of effect (Muir, 1985).

However, the sedatives in use with ketamine are associated with side effects that are of concern. Xylazine produces respiratory depression in most animals, and bradycardia, bradyarrhythmias and hypotension are observed depending on species and dose (Short, 1987). Diazepam is associated with species and occasional individual variability in 
response and its solvent (propylene glycol) may produce hypotension (Short, 1987). Acepromazine has prolonged duration of effect and is associated with hypotension secondary to marked peripheral vasodilation and response variability (Hall et al, 2001). Besides, rabbits have been reported to be the third most commonly anaesthetized species, but have at least seven times more risks of anaestheticrelated deaths compared to dogs and cats (Brodbelt, 2009). Inspite of the popularity of these three ketamine drug combinations, a comparative study of their relative efficacy and safety is yet to be reported in the rabbit.

The aim of this study, therefore, was to compare using rabbits the relative efficacy and safety of xylazine-ketamine (XK), diazepam-ketamine (DK) and acepromazine-ketamine (AK) at their respective intramuscular (im) doses recommended for clinical use. Selected anaesthetic indices and associated changes in heart rate (HR), respiratory rate (RR) and rectal temperature (RT) were determined at 10-min intervals in the first hour of anaesthesia.

\section{Materials and methods}

Experimental animals

10 apparently healthy adult New Zealand x American Chinchilla, rabbits of both sexes (four intact bucks and six intact does) weighing $1.8 \pm 0.2 \mathrm{~kg}$ (mean \pm sem) and acquired from the Experimental Animal Unit of the Faculty of Veterinary Medicine, University of Ibadan, Ibadan were used for the study. The rabbits were housed in pairs in cages and fed ad libitum mainly on commercial pelletized rabbit feed supplemented with freshly cut leaves of Tridax (Tridax procumbens), sweet potato (Ipomea batatas) and water leaf (Talinum trianuglare). Drinkable water was also provided ad libitum.

\section{Study protocol}

Each of the ten rabbits underwent three anaesthetic protocols (treatment) on three different occasions with one week interval allowed in between treatments. The anaesthetic protocols were designated as xylazine-ketamine (XK), acepromazineketamine (AK) and diazepam-ketamine (DK). Rabbits were premedicated the first time with xylazine (Chanazene ${ }^{\circledR}$, Chanelle laboratories $(20 \mathrm{mg} / \mathrm{ml}$ ) at a dose rate of $5 \mathrm{mg} / \mathrm{kg}$ body weight; the second time with diazepam (Valium 20 ${ }^{\circledR}$, Roche products Ltd, Welywn garden city, U.K, $5 \mathrm{mg} / \mathrm{ml}$ ) and the third time with acepromazine (Beruce ${ }^{\circledR}$, Berk pharmaceuticals Itd, Eastburne, England, $10 \mathrm{mg} / \mathrm{ml}$ ). Thirty minutes after premedication, each time anaesthesia was induced with ketamine (Non-proprietary; Rotexmedia Lab, Trittau Germany,50mg/ml) at a dose rate of $35 \mathrm{mg} / \mathrm{kg}$ when premedicated with xylazine (XK); $60 \mathrm{mg} / \mathrm{kg}$ when premedicated with diazepam (DK) and $75 \mathrm{mg} / \mathrm{kg}$ when premedicated with acepromazine (AK). All drugs were administered through the intrasmuscular route and dosages employed were as recommended by White \& Holmes (1976), Lipman et al (1990), and Wixison (1994). The time of induction was recorded.

Immediately after induction of anaesthesia, analgesia was assessed using the pedal withdrawal reflex. The hind paws were pinched with a pair of haemostatic forceps closed to the first ratchet at 2 min intervals and the time the rabbit was noticed not to withdraw the hind limbs in response to the pinch (loss of pedal reflex) was recorded. This pinching of the paws was continued at two minutes interval until the time when the rabbit started to withdraw the hind limbs in response to the paw pinch. This time was recorded as time of return of pedal reflex. Following the loss of the righting reflex by the anaesthetized rabbits, they were placed on right lateral recumbency on a padded wooden table.

\section{Calculated anaesthetic indices}

The following parameters were recorded and calculated as the anaesthetic indices:

a) Onset of loss of righting reflex (OLRR): Time interval (in $\mathrm{min}$ ) between the injection of ketamine and the loss of righting reflex by the rabbit.

b) Onset of analgesia (OA): Time interval (in min) between the injection of ketamine and the loss of pedal withdrawal reflex in both hind limbs by the anaesthetized rabbit.

c) Duration of analgesia (DA): Time interval (in min) between the loss and return of pedal withdrawal reflex in either of the hind limbs by the anaesthetized rabbit.

d) Duration at recumbency (DR): time interval (in min) between the loss of righting reflex and assumption of sternal posture by the anaesthetized rabbit.

e) Attempt to stand (ATS): Time interval (in min) between the assumption of sternal and standing postures by the anaesthetized rabbit.

\section{Measured physiological parameters}

In the course of each trial, heart rates (HR), respiratory rates (RR) and rectal temperature (RT) were determined immediately after the injection of ketamine, and thereafter, at 10 minutes intervals over the first 60 minutes of anaesthesia. Heart rate (in beats/min) was determined with the aid of a precordial stethoscope placed over the left second and fifth intercostal spaces, respiratory rate (in breaths / $\mathrm{min}$ ) was determined by observation of the rabbit's thoracic excursions and rectal temperature 
(in ${ }^{\circ} \mathrm{C}$ ) was determined using a digital clinical thermometer.

\section{Analysis of data}

All data recorded were calculated as means \pm standard error of means (sem) of 10 rabbits. The means of OLRR, OA, DA, DR and ATS for XK, DK and AK were compared using one way ANOVA followed by Bonferrom $t$ procedure when a significant difference was indicated. The mean values of the measured physiological parameters were compared using analysis of variance (ANOVA) for repeated measures followed as appropriate by Dunnett's test when a significant difference was indicated. A value of $P<0.05$ was considered statistically significant for all the comparisons (Dawson \& Trapps, 2004).

\section{Results}

The anaesthetic indices for the three drug combinations are shown in Table 1. OLRR did not differ significantly for the three drug combinations. Whereas DK and AK did not have any demonstrable analgesic effect, XK had OA of $4.2 \pm 0.1 \mathrm{~min}$ and DA of $57.0 \pm 0.7 \mathrm{~min} . \mathrm{DR}$ and ATS with $\mathrm{XK}$ of $62 \pm 1.5$ and $11.2 \pm 0.9$ were significantly shorter $(P<0.05)$ than with DK and AK of $184.4 \pm 3.3$ and 42.2 and of $169.6 \pm 3.0$ and $26.6 \pm 1.7$ respectively.

The mean HR, RR and RT responses of the rabbits that received $\mathrm{XK}, \mathrm{DK}$ and $\mathrm{AK}$ are compared in Figures 1,2 and 3 respectively.

Table 1: Anaesthetic indices of rabbits anaesthesized with intramuscularly administered ketamine following premedication with intramuscularly administered $x^{\prime}$ lazine ${ }^{a}$, diazepam ${ }^{a}$ or acepromazine ${ }^{c}$

\begin{tabular}{llll}
\hline Index & \multicolumn{3}{c}{ Treatment groups } \\
\cline { 2 - 4 } & XK & DK & AK \\
\hline OLRR(min) & $3.4 \pm 0.3$ & $2.0 \pm 0.0$ & $2.6 \pm 0.3$ \\
OA(min) & $4.2 \pm 0.1$ & NA & NA \\
DA(min) & $5.7 \pm 0.7$ & NA & NA \\
DR(min) & $62.0 \pm 1.5$ & $184.4 \pm 3.3$ & $169.6 \pm 0.3^{*}$ \\
ATS(min) & $11.2 \pm 0.9$ & $42.0 \pm 1.4$ & $26.6 \pm 1.7^{*}$ \\
\hline
\end{tabular}

Data were expressed as means \pm SEM of 10 rabbits

a- $5 \mathrm{mg} / \mathrm{kg}$ xylazine followed $30 \mathrm{~min}$ later by $35 \mathrm{mg} / \mathrm{kg}$ ketamine

b- $5 \mathrm{mg} / \mathrm{kg}$ diazepam followed $30 \mathrm{~min}$ later by $60 \mathrm{mg} / \mathrm{kg}$ ketamine

c- $5 \mathrm{mg} / \mathrm{kg}$ acepromazine followed $30 \mathrm{~min}$ later by $75 \mathrm{mg} / \mathrm{kg}$ ketamine

OLRR- Time to loss of righting reflex

OA- Onset of analgesia

DA- Duration of analgesia

DR- Duration at recumbency

ATS- Attempts to stand

NA- Not applicable

$* P<0.05$

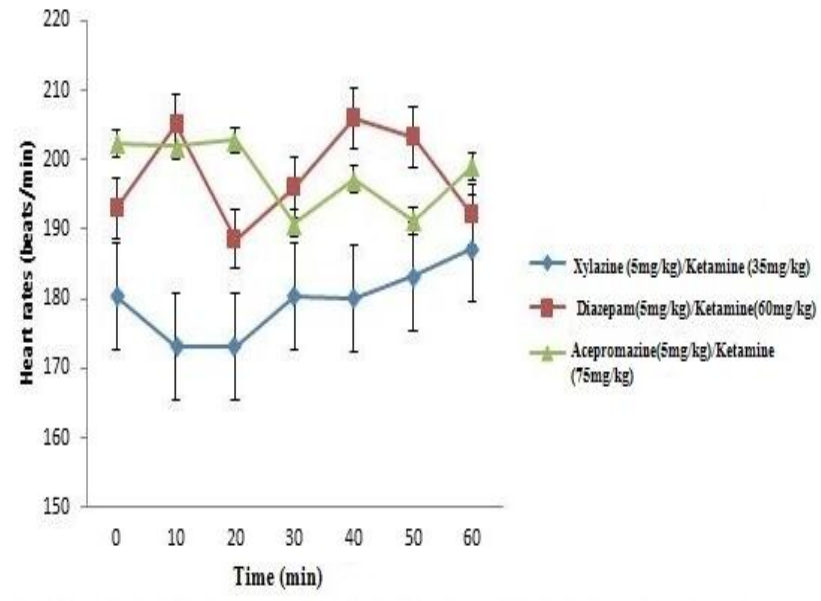

Figure 1: Heart rates of rabbits anaesthetized with intramuscularly administered ketamine following premedication with intramuscularly administered xylazine, diazepam or acepromazine

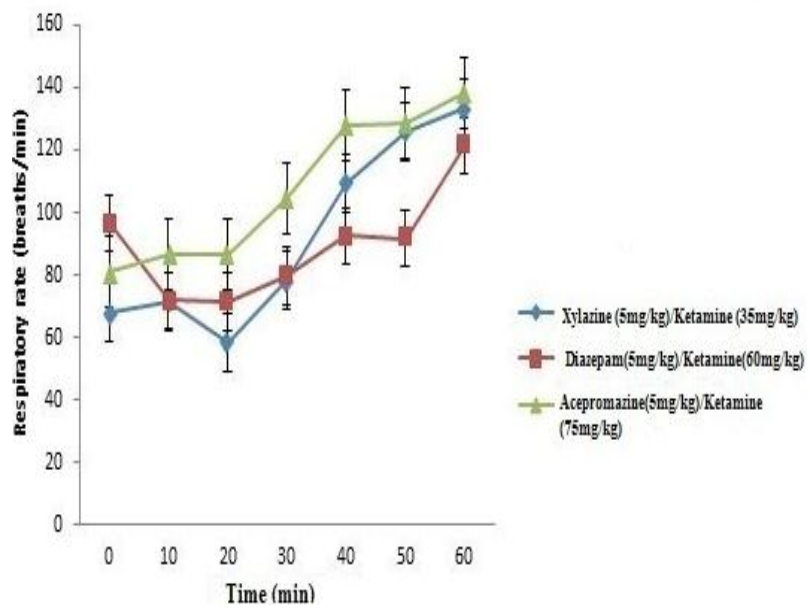

Figure 2: Respiratory rates of rabbits anaesthetized with intramuscularly administered ketamine following premedication with intramuscularly administered xylazine, diazepam or acepromazine 
Mean heart rate ranges were $173.2 \pm 6.6$ to $187.2 \pm 7.5 ; 188.6 \pm 6.4$ to $206.0 \pm 3.1$ and $190.8 \pm 1.3$ to $202.8 \pm 1.9$ beats/min with xylazine-ketamine, diazepam-ketamine, and acepromazine-ketamine respectively.

Mean respiratory rate ranges were $58.4 \pm 3.5$ to $133.0 \pm 11.2 ; 71.4 \pm 6.9$ to $121.2 \pm 12.6$ and $80.8 \pm 6.2$ to $137.0 \pm 4.3$ breaths/min with XK, DK and AK. Mean temperature ranges were $39.9 \pm 0.1$ to $40.0 \pm 0.1$; $39.0 \pm 0.3$ to $39.9 \pm 0.1$ and $39.6 \pm 0.1$ to $40.2 \pm 0.0^{\circ} \mathrm{C}$ with $\mathrm{XK}, \mathrm{DK}$ and $\mathrm{AK}$ respectively.

\section{Discussion}

Of the three drug combinations studied, only XK produced analgesia. This antinociceptive effect of XK cannot be ascribed to the action of the administered ketamine alone, since its use in combination with either diazepam or acepromazine even at respective higher doses of $60 \mathrm{mg} / \mathrm{kg}$ or $75 \mathrm{mg} / \mathrm{kg}$ than $35 \mathrm{mg} / \mathrm{kg}$ with xylazine, produced no demonstrable analgesic effect. Certainly, xylazine must have contributed to, and potentiated the analgesic action of ketamine in the drug combination.

The production of analgesia by XK in our study is consistent with findings of similar studies (White \& Holmes, 1976; Green et al, 1981; Lipman et al, 1990; Kazemi, 2002; Kilic, 2004). However, some workers despite using higher doses of xylazine-ketamine than the doses used in our study, reported lack of analgesia in all the rabbits studied (Amarpal et al, 2010) while others reported analgesia in only a few of the rabbits studied for only a short period (Henke et al, 2005). This low level of analgesia demonstrated by rabbits in these other studies compared with ours despite their use of higher dosages of xylazine-ketamine may be due to the breed and strain variations of the rabbits used. Indeed, it has been observed that rabbits display wide inter-individual variability in response to anaesthetics and show strain and sex specific differences (Avsaroglu et al., 2003).

The production of anaesthesia by XK in our study implies that in real clinical situation only XK is useful for painful procedures. Nonetheless, it may also be possible to extend the use of DK and AK to painful procedures with the addition of specific analgesic agents to them.

Our finding of lack of analgesia with the DK combination is in agreement with the findings of Green et al (1981) and Kazemi (2002) where DK produced good sedation and muscular relaxation but not enough analgesia to do surgery.

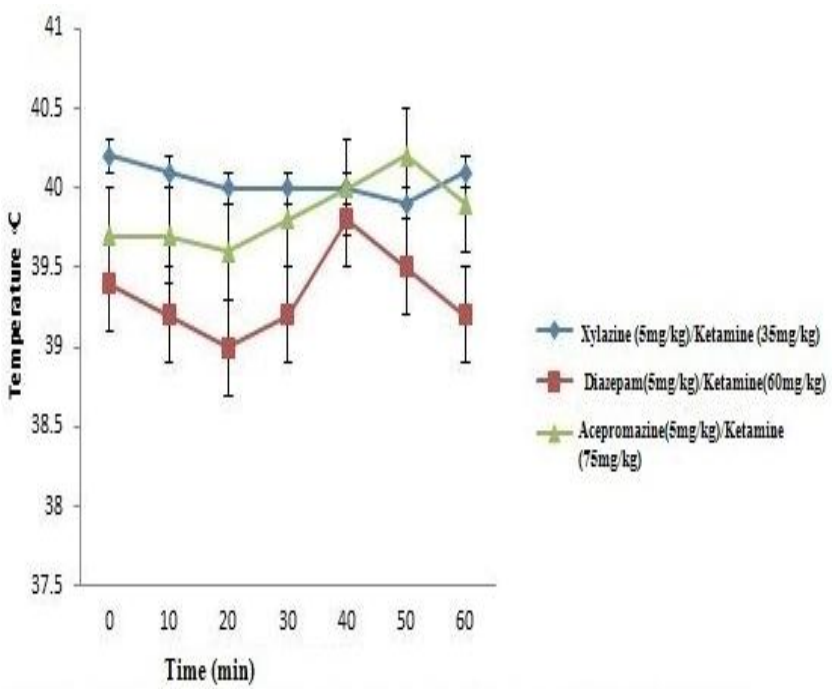

Figure 3: Rectal temperature of rabbits anaesthesized with intramuscularly administered ketamine following premedication with intramuscularly administered xylazine. Data are expressed as means \pm sem of 10 rabbits

Whereas in this study, AK did not produce analgesia, half of the rabbits given AK by Amarpal and others (2010) showed analgesia. This discrepancy may also be attributed to breed and strain variations of the rabbits used. However, as with our study, Kazemi (2002) also reported lack of analgesia with acepromazine- ketamine.

It is interesting to record longer duration of recumbency with both DK and AK than with XK. This finding may relate partly to the higher im doses of ketamine used in DK $(60 \mathrm{mg} / \mathrm{kg})$ and AK $(75 \mathrm{mg} / \mathrm{kg})$ than in XK $(35 \mathrm{mg} / \mathrm{kg})$. Prolonged muscle relaxing effects of diazepam and acepromazine, as well as the absence of surgical stimulation on the experimental rabbits, might also have contributed to the prolonged duration of recumbency.

The ranges of mean $\mathrm{HR}$ recorded for the three ketamine drug combinations are within the normal range of 130 to 325 beats / min accepted for the awake, resting rabbits (Harkness \& Wagner, 1995) making the anaesthetic regimes safe for clinical use. Mean RR associated with XK, DK and AK showed large variations as well as increases that could be described as polypnoea. The cause of the observed polypnoea in the anaesthetised rabbits is not known. The fast, panting breathing may reflect an attempt by the anaesthetised, recumbent rabbit to maintain minute volume or simply a response to hypercapnia and /or hypoxia. However, the latter supposition could not be confirmed as no blood gas analysis was 
carried out in the present study. Mean RT recorded for the three ketamine drug combinations are within the normal range of 38.5 to $40.0^{\circ} \mathrm{C}$ quoted for awake, resting rabbits (Harkness \& Wagner, 1995). In conclusion, all the three ketamine drug combinations produced unconsciousness and immobility, but only XK provided additional

\section{References}

Amarpal P, Kinjavdekar H, Aithal AM, Pawde JS \& Rahul U (2010). Evaluation of xylazine, acepromazine and medetomidine with ketamine for general anaesthesia in rabbits. Scandivanian Journal of Laboratory Animal Science, 37(3): 223-229.

Avsaroglu HA, Versluis L, Hellebrekers ZL, Haberham LF, Van Zutphen \& Van Lith HA (2003). Strain differences in response to propofol, ketamine and medetomidine in rabbits, Veterinary Records, 152(10): 300.

Brodbelt D (2009). Perioperative mortality in small animal anaesthesia. The Veterinary Journal, 182(2): 152-161.

Dawson B \& Trapps RG (2004). Basic and clinical biostatistics, 4th edition, McGraw Hill, New York. Pp 162-189.

Flecknell PA (2009). Laboratory animal anaesthesia, 3rd edition, Academic Press, London. Pp 6365.

Green CJ, Knight J \& Precious S (1981). Ketamine alone and combined with diazepam or xylazine in laboratory animals: a 10-year experience. Laboratory Animals, 15(2): 163170.

Hall LW, Clarke KW \& Trim CM (2001). Veterinary Anaesthesia, 10th edition, Elsevier, Oxford. Pp $75-112$.

Harkness JE \& Wagner JE (1995). The Rabbit. In: The Biology and Medicine of Rabbits and Rodents, 4th edition, Williams and Wilkins, Baltimore. Pp 13-30.

Henke J, Astner S, Brill T \& Eissner B(2005). Comparative study of three intramuscular anaesthetic combin ations (medetomidine/ketamine, analgesia. They were associated with normal HR and RT, as well as polypnoea of some clinical concern. Only XK is potentially useful for painful procedures. It may be possible to extend the use of DK and AK to painful procedures with the further addition of specific analgesic agents to them.

medetomidine/fentanyl/midazolam and xylazine/ketamine). Anaesthesia and Analgesia 32(3):261-270.

Kazemi D (2002). Evaluation of ketamine, xylazine, acepromazine and diazepam combinations for anaesthesia in rabbits. Indian Journal of Veterinary Surgery 23(1): 12-15.

Kilic N (2003). A comparison between Medetomidine-ketamine and Xylazineketamine anaesthesia in rabbits. Turkish Journal of Veterinary and Animal Sciences 28:921-926.

Lipman NS, Marini RP \& Erdman SE (1990). A comparison of ketamine/xylazine and ketamine/xylazine/ acepromazine anesthesia in the rabbit. Laboratory Animal Science, 40(4): 395-398.

Muir WW (1985). Cylohexamine drug mixtures: The pharmacology of ketamine and ketamine drug combinations. Proceedings of second International Congress of Veterinary Anesthesia. Veterinary Publishing Co, Santa Barbara. Pp 5-14.

Short CE (1987). Principles and Practice of Veterinary Anesthesia. Williams \& Wilkins, Baltimore Pp 158-169.

White GL \& Holmes DD (1976). A comparison of ketamine and the combination ketamine xylazine for effective surgical anesthesia in the rabbit. Laboratory Animal Science, 26(5): 804-806.

Wixison SK (1994). Anesthesia and analgesia. In: The Biology of the Laboratory Rabbit, 2nd edition, (Maning PJ, Ringler DH \& Newcomer $C E$, editors), Academic Press, San Diego. Pp 87-109. 